\title{
A Power-Controlled MAC Supporting Service Differentiation in Mobile Ad Hoc Networks
}

\author{
Wenjian Shao, Dongxu Shen, Daiqin Yang and Victor O. K. Li \\ Department of Electrical and Electronic Engineering \\ The University of Hong Kong \\ Pokfulam, Hong Kong, China \\ \{wjshao, dxshen, dqyang, vli\}@eee.hku.hk
}

\begin{abstract}
The original power controlled multiple access (PCMA) protocol does not support service differentiation. In this paper, we extend PCMA to form a new media access control protocol supporting service differentiation in mobile ad hoc networks. To support QoS, we first introduce the in-station access category concept in 802.11e to PCMA. For service differentiation between access categories, our major contribution is to propose a senderinitiated busy tone based mechanism that allows a user to gain quick channel access. This quick access mechanism is only performed when the number of access failures exceeds a threshold. An access category with higher priority is assigned a lower threshold for easier channel access, and vice versa. Through analysis and simulation, we demonstrate that our protocol can provide better quality of service than 802.11e in terms of throughput, delay, loss, and fairness.
\end{abstract}

\section{INTRODUCTION}

The provisioning of quality of service $(\mathrm{QoS})$ is central to the success of mobile ad hoc networks (MANET). Basically, there are two strategies for QoS support. Integrated services (IntServ) guarantees QoS requirements such as bandwidth and delay, but it requires complicated schemes for resource reservation and allocation. A simpler approach is differentiated services (DiffServ), which prioritizes one type of traffic over another, with no "hard" guarantees. In the dynamic environment of MANET, DiffServ is easy to implement, because each hop operates independently without end-to-end resource reservation. In this paper, we discuss the design of power-controlled media access control (MAC) protocol that supports DiffServ in MANET.

The achievement of DiffServ in MANET relies on the design of the MAC protocol. High priority traffic should have precedence over low priority traffic in accessing the channel. Using this approach, [2], [3], [4] and [5] have been proposed to provide service differentiation in IEEE 802.11 [1] based MANET. Also, the developing IEEE 802.1 le [6] MAC protocol supports DiffServ in this style. A major drawback of 802.11 based protocols is that the transmission power is fixed, thus leading to low channel reuse ratio.

Power-controlled MAC protocols, such as PCMA [7], can effectively increase channel reuse by adjusting the transmission power to an optimal value instead of a fixed one. However, PCMA does not consider QoS. In this paper, we provide DiffServ support in PCMA, called PCMA-DS(DiffServ).

First, we introduce the access category (AC) concept in 802.1 le to PCMA. Our major contribution is to incorporate a sender-initiated busy tone with PCMA to differentiate the channel access probabilities between different access categories. In our protocol, the sender-initiated busy tone is used to assist in channel access. When a user is in urgent need of channel access, for example, it has a delay-sensitive packet to send, it can turn on the busy tone as a declaration. Overhearing the busy tone, nearby nodes will refrain from transmission to yield the access right to the urgent user. A high priority traffic is allowed to apply the above busy tone mechanism earlier than low priority traffic. Using computer simulation, we compare our protocol with IEEE 802.11e and PCMA. We find our protocol perform better than 802.1 le and PCMA in terms of throughput, delay, packet loss, and fairness. Since we focus on extending PCMA to support DiffServ, we do not consider the energy consumption. This issue has been discussed in many papers, such as [8] and [10].

This paper is organized as follows. In Section II, we review the related work. In Section III, we describe the MAC protocol. Simulation results are provided in Section IV. Conclusion is given in Section V.

\section{RELATED WORK}

\section{A. Differentiated Services in 802.11 Based Protocols}

The basic mode of 802.11 [1] is the Distributed Coordination Function (DCF), which uses CSMA/CA as the fundamental channel access scheme. There are two basic mechanisms in CSMA/CA for collision avoidance: inter-frame space (IFS) and backoff. When a station wants to transmit a packet, it senses the channel first and defers the transmission for predefined IFS even if the channel is sensed idle. If the channel is detected busy, a backoff procedure is invoked, with the backoff time decided as rand $(0, C W) \cdot a$ Slot_time, where aSlot_time is the slot time. The function $\operatorname{rand}(0, C W)$ picks a value randomly from the range $[0, C W]$, where $C W$ is the contention window size. QoS is not supported in 802.11 DCF. Although the optional polling-based scheme (PCF) of 802.11 is designed for time-bounded services in wireless LAN, it has many limitations. More importantly, PCF only works for the infrastructure configuration, not for the ad hoc mode.

Service differentiation can be achieved in 802.11 DCF by modifying the IFS (based on different IFS values [2][3]) and backoff times (based on the control of $C W$ [3][4] or based on the selection of different backoff times directly [5]). Smaller 
IFS and backoff time result in higher priority. These methods provide only one priority for one station, which is not the case in actual networks.

IEEE 802.11e extends the 802.11 MAC with a new Hybrid Coordination Function (HCF). HCF combines two methods, i.e. the enhanced distributed channel access scheme (EDCA) and the polling-based HCF controlled channel access scheme (HCCA). HCCA is the enhancement of PCF and not suitable for infrastructureless MANET. In the environment of MANET, we consider the distributed EDCA. EDCA is the extension of DCF to support DiffServ. By introducing in-station access categories (AC), a QoS station can support four delivery priorities, and eight user-defined traffic categories (TC) are mapped to four ACs. Each AC has its independent queue and behaves as an independent MAC to contend for the channel. Since each $\mathrm{AC}$ is controlled by predefined parameters, such as arbitration IFS (AIFS), $C W_{\min }$ and $C W_{\max }$, priorities are implemented between ACs.

\section{B. Power-Controlled MAC Protocol}

A major drawback of IEEE 802.11 based MAC protocols is that the transmission power is always fixed, regardless of communication distance. This feature makes 802.11 based protocols inefficient in channel reuse. To improve the channel utilization, a group of protocols using the power control mechanism are proposed. The transmission power in these protocols is adjusted to an optimal value instead of a fixed value, thus reducing interference and encouraging channel reuse.

Among power-controlled MAC protocols, PCMA [7] is a notable one. In PCMA, each node needs to calculate the maximum allowable power for its transmission without interfering with other ongoing receptions. This calculation technique is called power bounding. The power bound $\left(P t_{\text {bound }}\right)$ is designed such that after propagation loss, the received power will not exceed the tolerable additional noise $\left(N_{a}\right)$ of all neighboring receivers. To announce its tolerable additional noise, each receiver adopts a receiver-initiated power-controlled busy tone $\left(B T_{r}\right)$, whose transmission power $\left(P t_{B T r}\right)$ is inversely proportional to its $N_{a}$. Periodical pulses instead of continuous signals are used for busy tones so that tones from different nodes can be distinguished. According to the maximal received power on $B T_{r}$ channel $\left(P r_{B T r}\right)$, each node can identify the most vulnerable receiver around it and decide its $P t_{\text {bound }}$. In PCMA, a transmitter sends a request using its $P t_{\text {bound }}$. After receiving the request, the receiver calculates the desired power $\left(P t_{d e s}\right)$ and $N_{a}$ for the transmission according to the channel gain and local noise level. If $P t_{\text {bound }} \geq P t_{\text {des }}$, the receiver accepts the request and data are sent with $P t_{d e s}$.

PCMA uses power-controlled receiver busy tone and power bounding algorithm to solve the hidden/exposed terminal problems and increase channel reuse. The power-controlled receiver busy tone signifies an ongoing reception, thus solving the hidden terminal problem. IEEE 802.11 [1] makes access decision based on the channel status, i.e., whether the channel is free or busy. Unlike the "on/off model" used by IEEE 802.11, PCMA allows transmitters to access the channel even when the channel is busy, as long as the transmission power is lower than $P t_{\text {bound }}$, and the exposed terminal problem is implicitly solved.
Therefore, PCMA can achieve much higher throughput than 802.11 based MAC protocol, especially at high load.

\section{Supporting DiffSERV In PCMA}

\section{A. Design Motivations}

To support DiffServ in PCMA, we can borrow the concept of access categories from 802.11e to PCMA. For different ACs, a mechanism is needed to differentiate the channel access probability. In 802.11 e, this differentiation is achieved through different IFSs and backoff times. The AC with smaller AIFS and backoff time (controlled by $C W_{\min }$ and $C W_{\max }$ ) is more likely to win the contention. It should be noticed that the operations of IFS and backoff time are conditioned on channel status, i.e., whether the channel is busy or free.

However, as discussed previously, the transmission decision of PCMA is not related to whether the channel is busy: a station is allowed to transmit as long as the desired transmission power satisfies the transmission power bound, regardless of the channel status. In PCMA, a station will refrain from transmission when its power bound is less than its desired transmission power, i.e., $P t_{\text {Bound }}<P t_{\text {des }}$. With small IFS and backoff time, a station can have a quick retry. However, its power bound may still be less than its desired transmission power on the retry. On the contrary, a station with large IFS and backoff time may have $P t_{\text {Bound }} \geq P t_{\text {des }}$, thus successfully accessing the channel. Therefore, control of IFS and backoff time is not an effective way to implement DiffServ in PCMA.

Obviously, the channel access probability is determined by $\operatorname{Pr}\left\{P t_{\text {Bound }} \geq P t_{\text {des }}\right\}$. If it is easier for the transmission power bound of a station to meet the requirement of desired power than other stations, this station is more like to access the channel and has higher priority. Since $P t_{d e s}$ is determined by the communication distance, $S N R$ requirement, and receiver noise level, we can only manipulate $P t_{B o u n d}$ to achieve DiffServ. The value of $P t_{B o u n d}$ is dependent on the on-going transmissions within the vicinity. If there are more transmissions around, $P t_{\text {Bound }}$ will become smaller, and vice versa. Different stations have different $P t_{\text {Bound }}$. Even for a single station, its $P t_{\text {Bound }}$ is time varying.

Ideally, if $P t_{B o u n d}$ can monotonically increase after a station has decided to transmit, channel access can be quickly obtained. This is possible if the neighboring nodes cooperate in the following way: neighboring nodes that are in the idle state do not compete for transmission, and neighboring nodes in transmission will not initiate new transmission after they finish. In this way, interference to the intending node will subside and its $P t_{\text {Bound }}$ will increase. Once $P t_{\text {Bound }}$ rises above $P t_{d e s}$, the node can start transmission.

The above is only possible when the neighboring nodes are aware of the transmission intention of that station. In our protocol, we devise a declaration mechanism assisted by a senderinitiated power-controlled busy tone for quick channel access. In such a scheme, when a node fails to access the channel since its power bound is smaller than the desired transmission power, it will turn on the sender-initiated busy tone using a properly selected power. Overhearing the busy tone, nearby nodes will refrain from transmissions to yield the access right to the urgent 
node. For service differentiation, high priority traffic is allowed to apply the sender-initiated busy tone mechanism earlier than low priority traffic.

\section{B. System Assumptions}

In this paper, we make the same assumptions on the channels as in PCMA [7]. They are:

- The channel gain remains unchanged during the transmission of RTS and DATA.

- The channel gain from node A to node B equals the channel gain from node B to node $A$.

- The gain of the data channel equals the gain of the busy tone channel.

For the same considerations, periodical busy tone pulses instead of a solid tone are used in the busy tone channel as in PCMA. According to [9], only 1-2\% of bandwidth is needed for busy tones to achieve the best performance of the system. Thus, we assume the overhead caused by busy tones is negligible.

We also assume nodes know the required transmission power to the desired location (denoted as $P t_{d e s}$ ). To determine $P t_{d e s}$, a sender must have the distance information between itself and the receiver. Many location-aware MAC protocols are proposed to help senders determine the distance to the desired destination, for example, through global positioning system (GPS). Distance also can be estimated through the previous transmissions, as discussed in [10].

\section{Protocol Description}

1) Quick Channel Access by Busy Tone Declaration: The basic idea of the quick channel access is to use a sender-initiated power-controlled busy tone as a mechanism to announce the access request. We denote this busy tone as $B T_{t}$, and the receiverinitiated busy tone in PCMA as $B T_{r}$. An intending node will set up $B T_{t}$ using power $P t_{\text {des }}$ to notify its neighbors that it wants to send packets using this power. Nodes overhearing the busy tone will update their local noise level accordingly, and those that may otherwise transmit will refrain from transmitting. Gradually, with the completion of on-going transmissions, $P t_{\text {Bound }}$ will become larger than $P t_{d e s}$, after which the node can transmit.

The busy tone declaration steps are summarized as follows.

1) When node A fails to access the channel for more than a given threshold (represented as $F \_T h r e s h$ ) number of times, it turns on $B T_{t}$ using $P t_{d e s}$ until it can access the channel.

2) If a node senses the $B T_{t}$ channel with maximal received power $\left(P r_{B T t}\right)$ larger than the threshold for correct packet decoding ( $R X_{-}$Thresh), i.e., $\operatorname{Pr}_{B T t} \geq$ $R X_{\text {_T }}$ Thesh, it will both backoff its transmission and reject any RTS request until this periodical pulse disappears.

3) Else, a node will add the received pulse power to its noise level on the data channel to account for the interference from node $\mathrm{A}$ when node $\mathrm{A}$ transmits later.

4) After node A turns on its $B T_{t}$, it continues sensing the $B T_{r}$ channel and updating its $P t_{\text {bound }}$. When $P t_{\text {bound }} \geq$
$P t_{d e s}$, it turns off its $B T_{t}$ and tries to access the channel using the steps in PCMA.

Obviously, this scheme assures prompt channel access. In our protocol, since nodes sensing $P r_{B T t} \geq R X \_T h r e s h$ are potential receivers of node $A$, they will refrain from new communications (both send and receive) and wait for A's connection request. With the completions of ongoing receptions around node $\mathrm{A}, \mathrm{Pt} t_{\text {bound }}$ of node A gradually increases. When it becomes greater than $P t_{d e s}$, node $\mathrm{A}$ is eligible to access the channel. On the other hand, because nodes sensing $\operatorname{Pr}_{B T t}<$ $R X \_T h r e s h$ will not be the receivers of node A, they can continue setting up new connections. Their transmissions will not collide with the later transmission from node $\mathrm{A}$, since interference from node $\mathrm{A}$ has been considered.

We incorporate the sender-initiated busy tone with PCMA to generate a dual busy tone MAC protocol. So, a node in idle status not only monitors the $B T_{r}$ channel to update its power bound, it also monitors the $B T_{t}$ channel to check whether it is blocked by the $B T_{t}$ signal. Usually, a node will access the channel the same way as in PCMA, except it needs to monitor the $B T_{t}$ channel. If it fails, it will backoff and access again. Only when the number of failures reaches a predefined threshold, $F_{-}$Thresh, will the node use the $B T_{t}$ as an assistance to access the channel.

2) Integration of Access Categories in PCMA: In our protocol, we use the method similar to that in 802.11e to support multiple access categories inside one station. There are multiple independent queues in our MAC protocol, and each AC maintains its own queue. The traffic will be mapped to these queues according to the traffic type. Each $\mathrm{AC}$ independently contends for the channel using the steps defined in PCMA as a virtual station. If more than two ACs within a station happen to contend the channel at the same time, a virtual collision occurs. In this situation, the $\mathrm{AC}$ with higher priority will win the virtual contention. When an $\mathrm{AC}$ fails to access the channel, including virtual collision, it will backoff and try again.

Note that all ACs have the same $C W_{\min }$ and $C W_{\max }$ in our protocol. We do not differentiate the ACs according to backoff times. We differentiate ACs by assigning different $F_{-}$Thresh values to different ACs. A smaller $F \_$Thresh favors higher priority $\mathrm{AC}$, which will use the busy tone declaration mechanism earlier and have fewer access attempts than lower priority AC.

\section{Simulation Results}

In our simulation, we study the DiffServ capability of our protocol using NS2.27, and compare it with that of both 802.11e EDCA and PCMA.

\section{A. Simulation Setup}

In the test environment, we use the default settings of wireless channel and physical layer in NS2.27. The antenna gain is 1 , the height of each antenna is $1.5 \mathrm{~m}$, the system loss is 1 and the carrier frequency is $916 \mathrm{MHz}$. The $R X \_T h r e s h$ is $-64 \mathrm{dBm}, C S \_$Thresh is $-78 \mathrm{dBm}, S I R \_$Thresh is $10 \mathrm{~dB}$ and the maximal transmission power is $24.5 \mathrm{dBm}$. For propagation model, two-ray-ground model is used. With these settings, the maximal transmission range is $250 \mathrm{~m}$. 
We construct a network with an area of $1000 \mathrm{~m} \times 1000 \mathrm{~m}$, within which 100 nodes are randomly distributed. The bandwidth of the data channel is $2 \mathrm{Mbps}$. The maximal queue length for each $\mathrm{AC}$ is 50 packets. In this paper, since we focus on the performance of the MAC layer protocol, only one hop neighbors are randomly selected to form communication pairs so as to remove the influence of routing. In future research, we will study the performance of our protocol under multi-hop environment.

Three types of traffic are transmitted between each communication pair. The first traffic type is the background traffic following the Poisson traffic model. Its average data rate is 40 Kbps. It has the lowest priority and is mapped to AC_BK. The second type is MPEG1 video traffic with an intermediate priority. It is VBR with an average rate of $32 \mathrm{Kbps}$ and mapped to AC_VI. The third type is real time voice traffic, which has the highest priority. The voice traffic is CBR at $8 \mathrm{Kbps}$ and mapped to AC_VO. The parameters of ACs are configured in Table 1, where $C W_{\min }, C W_{\max }$ and $A I F S N$ are the parameters for 802.11e and F_Thresh is for our protocol. The F_Thresh value of $\mathrm{AC}_{-} \mathrm{BK}$ is "N/A" means that the quick channel access method is not available for AC_BK. When increasing the number of communication pairs from 10 to 100 , the normalized (by the channel bandwidth) traffic load $G$ is from 0.4 to 4 .

Table 1: Configuration of access categories

\begin{tabular}{|c|c|c|c||c|}
\hline AC & $C W_{\min }$ & $C W_{\max }$ & $A I F S N$ & $F_{\text {_Thresh }}$ \\
\hline $\mathrm{AC} \_V O$ & 3 & 7 & 2 & 1 \\
\hline $\mathrm{AC} \_V I$ & 7 & 15 & 2 & 2 \\
\hline AC_BK & 15 & 1023 & 7 & N/A \\
\hline
\end{tabular}

\section{B. Performance Comparison}

1) Throughput: We first compare the throughput of our PCMA-SD protocol with 802.11e EDCA and the original PCMA. Fig. 1 shows the average throughput of three types of

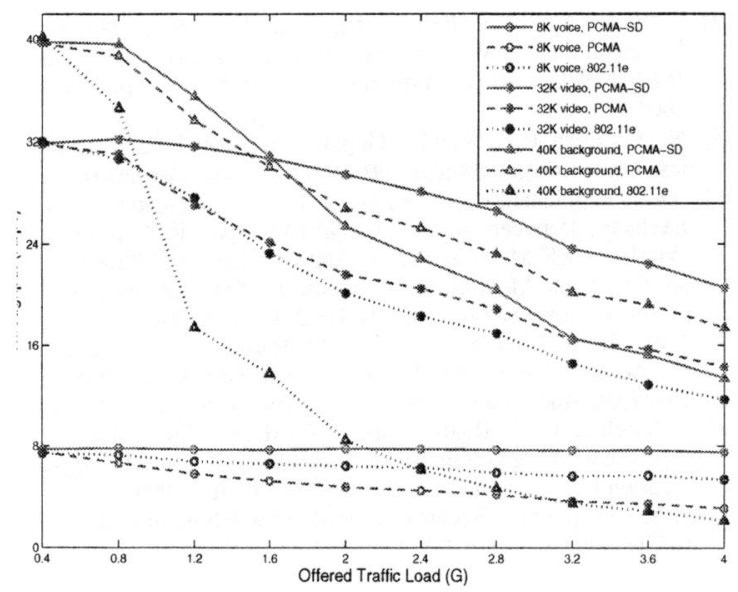

Fig. 1. Throughput comparisons of three types of traffic among 802.11 e EDCA, PCMA and PCMA-SD.

traffic for one communication pair. It represents the average throughput a user can obtain for each type of traffic. Firstly, we observe that our protocol can provide DiffServ support. For high priority voice and video traffic, our protocol can provide much higher throughput than PCMA. While the performance of low priority background traffic is worse than PCMA since the aggregated throughput of our protocol is almost the same as that of PCMA.

Secondly, we observe that our protocol can provide better differentiated services than 802.11e EDCA. As load increasing, the throughput of the three types of traffic decline with different rates according to their priorities. Since our protocol uses a power control mechanism, thus enjoying a higher channel reuse than $802.11 \mathrm{e}$, the degradation is much less severe. More importantly, voice traffic, with the highest priority, exhibits a constant throughput in our protocol. In contrast, voice throughput in 802.11 e reduces by $30 \%$ when the traffic load $G=4$.

Thirdly, we observe that PCMA has the worst performance in voice traffic throughput, since it has no DiffServ support. However, since PCMA has higher channel reuse ratio and higher aggregated throughput than $802.11 \mathrm{e}$, it outperforms 802.11 e with medium priority video and low priority background traffic. We can say that the capacity of a MAC protocol is a key to QoS support.
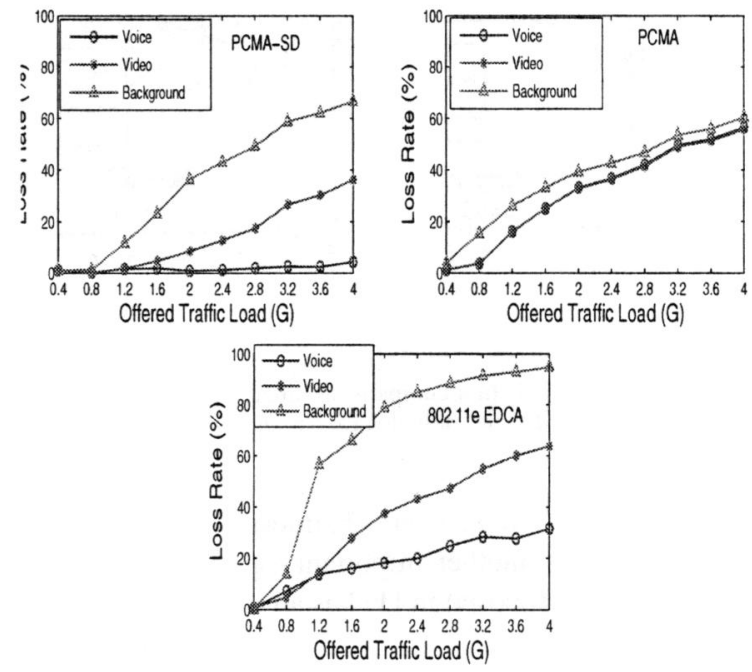

Fig. 2. Loss rate comparisons of three types of traffic among 802.11e EDCA, PCMA and PCMA-SD.

2) Loss Rate: Fig. 2 compares the loss rates, another important QoS measure. From Fig. 2, we can see that three types of traffic experience different loss rates in our protocol, while the loss rates are almost the same in PCMA, which is another indication of DiffServ capability of our protocol.

We also find that our protocol achieves lower loss rates than 802.11e EDCA for all three traffic types. Especially, the loss rate of the highest priority voice traffic is near zero in our protocol.

3) Delay: Fig. 3 presents the distribution of end-to-end delay under different traffic loads. For delay-sensitive applications, such as voice and video, both 802.11e EDCA and our 
protocol can provide small end-to-end delay at the sacrifice of background traffic. Since PCMA can not provide DiffServ, all traffic experience the same delay. With the increase of traffic load ( $G$ from 0.8 to 1.6 ), the delay also increases. However, at all loads, our protocol has consistently better delay performance than $802.11 \mathrm{e}$.
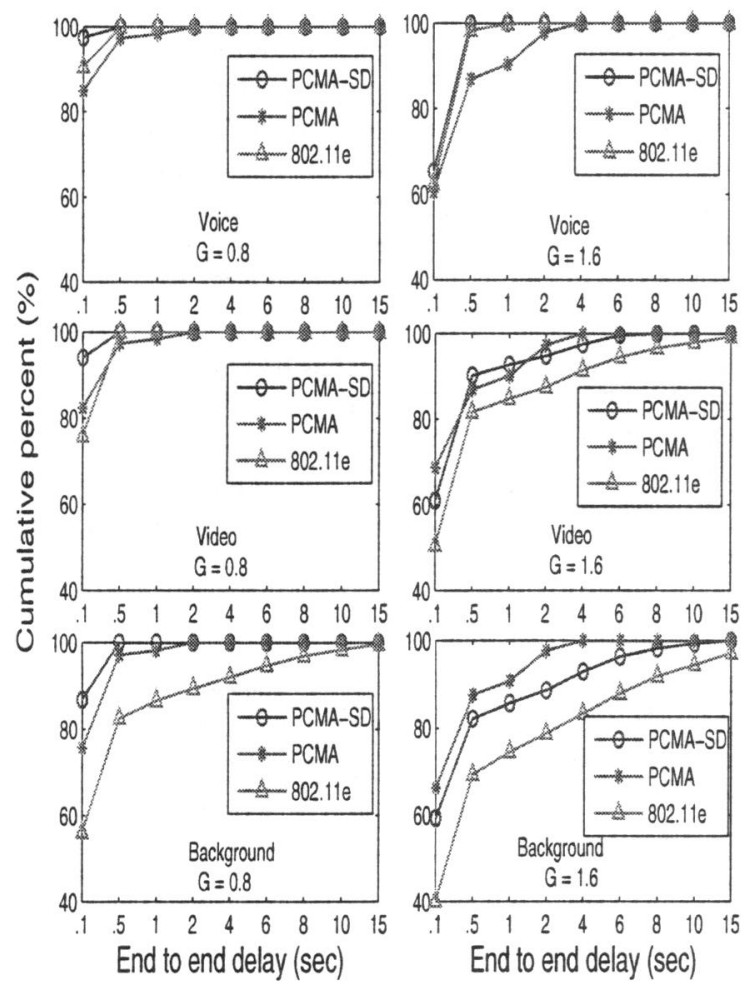

Fig. 3. Delay distribution comparisons among 802.11e EDCA, PCMA and PCMA-SD under different traffic load ( $G=0.8$ and 1.6).

4) Fairness Comparison: Fairness between flows with the same priority is another important aspect in QoS. We use the fairness index proposed in [11] as an evaluation metric.

$$
\text { fairness index }=\frac{\left(\sum_{i=1}^{n} T_{i}\right)^{2}}{n * \sum_{i=1}^{n}\left(T_{i}\right)^{2}}
$$

where $n$ is the total number of flows having the same priority and $T_{i}$ is the throughput of a flow. It is most fair when the fairness index is 1 , and less fair with lower index values.

Fig. 4 shows the fairness indices of the three types of traffic. The fairness of our protocol is much better than 802.11e for all traffic types, because the fairness index exhibits little degradation with respect to the overall load.

\section{CONCLUSION}

In this paper, we propose a power-controlled MAC protocol to support service differentiation in ad hoc networks. We have two contributions in this paper. First, we incorporate the access category concept into PCMA. Second and the major contribution is that we design a sender-initiated power-controlled
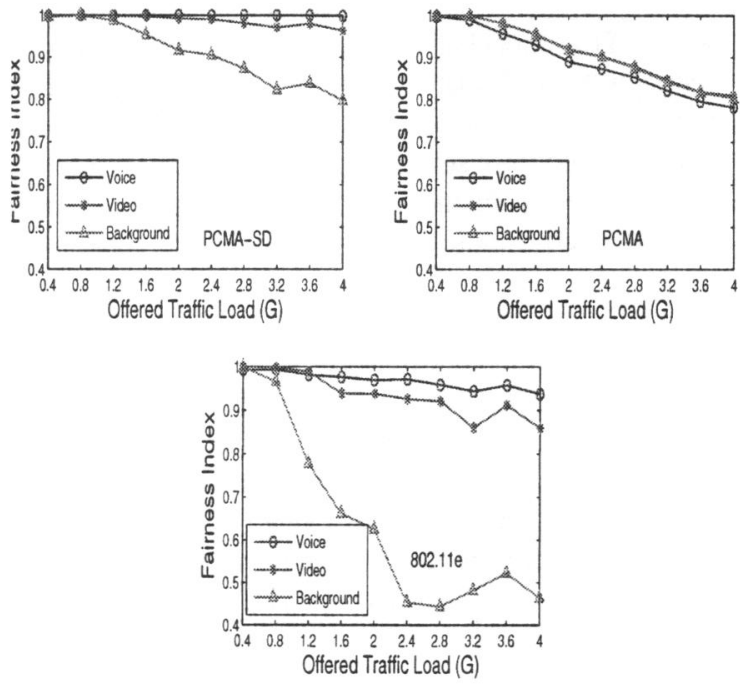

Fig. 4. Fairness index comparisons among 802.11e EDCA, PCMA and PCMA-SD.

busy tone declaration scheme for quick channel access, based on which service differentiation can be achieved. Simulation results show that our protocol can provide service differentiation and achieve better throughput, loss, and delay performance than $802.11 \mathrm{e}$ EDCA, and better fairness at the same time.

\section{REFERENCES}

[1] ANSI/IEEE Std 802.11. 1999 Edn. Part 11: Wireless LAN Medium Access Control (MAC) and Physical Layer (PHY) specifications.

[2] D-J. Deng and R-S. Chang. "A Priority Scheme for IEEE 802.11 DCF Access Method," IEICE Transactions on Communications, Vol.E82-B, No.1, pp. 96-102, Jan. 1999.

[3] I. Aad and C. Castelluccia. "Differentiation Methanisms for IEEE 802.11," Proc. IEEE INFOCOM*01, Anchorage. Alaska, USA. Apr. 2001, pp. 209218.

[4] A. Veres, A. T. Campbell, M. Barry and L. H. Sun, "Supporting Service Differentiation in Wireless Packet Networks Using Distributed Control," IEEE J. Select. Areas Commun., Vol. 19, No. 10, pp. 2094-2104, Oct. 2001.

[5] N. H. Vaidya, P. Bahl and S. Gupta, "Distributed Fair Scheduling in Wireless LAN," Proc. Mobicom'00, Boston. USA, Aug. 2000, pp. 167-178.

[6] Draft Supplement to Standard for Telecommunications and Information Exchange Between Systems-LAN/MAN Specific Requirements - Part11: Wireless LAN Medium Access Control (MAC) and Physical Layer (PHY) specifications: Medium Access Control (MAC) Enhancements for Quality of Service (QoS), IEEE 802.11e/Draft 4.2. Feb. 2003.

[7] J. Monks, V. Bharghavan and W.-M. Hwu, "A Power Controlled Multiple Access Protocol for Wireless Packet Networks." Proc. IEEE INFOCOM'01. Anchorage. Alaska. USA. Apr. 2001, pp. 219-228.

[8] S. Singh and C.S. Raghavendra. "PAMAS - Power Aware Multi-access Protocol with Signalling for Ad Hoc Networks," ACM Computer Communication Review, Vol. 28. Issue 3. pp. 5-26. Jul. 1998.

[9] F. A. Tobagi and L. Kleinrock. "Packet Switching in Radio Channels: Part II-The Hidden Terminal Problem in Carrier Sense Multiple-Access and the Busy-Tone Solution." IEEE Trans. Commun., Vol. COM-23, No. 12. pp. 1417-1433. Dec. 1975.

[10] J. Gomez, A. T. Campbell, M. Naghshineh and C. Bisdikian, "PARO: Supporting Dynamic Power Controlled Routing in Wireless Ad Hoc Networks," ACM/Kluwer Journal on Wireless Networks(WINET), Vol. 9, Issue 5. pp. 443-460, Sept. 2003.

[11] R. Jains. The Art of Computer Systems Performance Analysis, John Wiley \& Sons, 1991. 